\title{
PERSPECTIVAS LATINOAMERICANAS SOBRE MEDICINA CENTRADA EN LA PERSONA
}

\author{
Alberto Perales ${ }^{1, a}$, Juan Mendoza ${ }^{2,3,4, b}$, Rodolfo Armas ${ }^{5, c}$, Oscar Cluzett,d
}

\begin{abstract}
RESUMEN
La medicina centrada en la persona (MCP) es un movimiento programático mundial liderado por el International College of Person Centered Medicine. Su ingreso en Latinoamérica es reciente. Reclama el empleo de la investigación científica como instrumento para generar la mejor evidencia clínica, y el humanismo como esencia de la medicina en bien de la humanidad. Su propuesta no es solo combatir la enfermedad, sino, también, promover el despliegue de los potenciales saludables del ser humano hacia el logro de su bienestar y desarrollo integral. Aunque el humanismo de la medicina en Latinoamérica ha sido característica distintiva de su práctica, se aprecia en épocas actuales una preocupante reducción de su influencia en la atención de salud por factores diversos. El presente artículo compendia perspectivas latinoamericanas de cuatro países. Se cotejan necesidades y experiencias y describen respuestas que vienen generándose frente a la influencia deshumanizadora de la tecnología y del manejo de la salud como bien de consumo.
\end{abstract}

Palabras clave: Medicina Centrada en la Persona -Sistemas de Atención de Salud- - Educación Médica- Investigación en Salud - Salud Pública en Latinoamérica (fuente: DeCS BIREME).

\section{LATIN AMERICAN PERSPECTIVES ON PERSON-CENTERED MEDICINE}

\begin{abstract}
Person-centered medicine (PCM) is a programmatic global initiative led by the International College of Person-Centered Medicine. It has recently emerged in Latin America. It requires the use of scientific research as an instrument to generate the best clinical evidence, and humanism as the essence of medicine to help mankind. It is focused on not only combatting disease but also promoting the display of healthy human being potentials towards achieving well-being and comprehensive growth. Although the humanism of medicine in Latin America has been a distinctive characteristic of its practice, now, there is a worrying decline in its impact on healthcare. This article summarizes the Latin American perspective from four countries. Needs and experiences are compared and responses that arise in view of the dehumanizing influence of technology and health management as a consumption good are described.
\end{abstract}

Key words: Person Centered Medicine -Health Care Systems- - Medical Education - Health Research - Public Health in Latin America (Source: DeCS BIREME).

\section{INTRODUCCCIÓN}

Juan Enrique Mezzich y sus colaboradores del International College of Person Centered Medicine, han descrito los ocho principios de la medicina centrada en la persona (MCP) ${ }^{(1)}$ : compromiso ético, marco holístico, sensibilidad y respuesta cultural; foco comunicativo y relacional; individualización del programa de atención clínica; establecer una base común entre clínicos, paciente y familia para el entendimiento y la acción; organización de servicios integrados y centrados en las personas y la comunidad; educación médica e investigación científica en salud centradas en la persona. A esta perspectiva general se suman aspectos particulares ligados a la realidad de cada país. A continuación, se presentan algunas reflexiones sobre la MCP desde la perspectiva de cuatro países latinoamericanos.

\footnotetext{
Facultad de Medicina, Instituto de Ética en Salud, Universidad Nacional Mayor de San Marcos. Lima, Perú.

Universidad Nacional de Colombia. Colombia.

Academia Nacional de Medicina de Colombia. Colombia.

Facultad de Medicina, Colegio Mayor de Nuestra Señora del Rosario. Bogotá, Colombia.

Universidad de Chile. Chile

Academia Nacional de Medicina de Uruguay. Uruguay

a Médico psiquiatra. Doctor en Medicina; ${ }^{b}$ Médico neurocirujano. Doctor en Medicina y Cirugía; ${ }^{\mathrm{c}}$ Médico gastroenterólogo; ${ }^{\mathrm{d}}$ Médico. Postgrado en cirugía, medicina intensiva y emergencia. Magister en Bioética.

Recibido: 04/11/2016 Aprobado: 23/11/2016
}

Citar como: Perales A, Mendoza J, Armas R, Cluzet O. Perspectivas latinoamericanas sobre medicina centrada en la persona. Rev Peru Med Exp Salud Publica. 2016;33(4):801-10. doi:10.17843/rpmesp.2016.334.2568 


\section{REFLEXIONES SOBRE LA MEDICINA CENTRADA EN LA PERSONA DESDE LA PERSPECTIVA PERUANA}

Pedro Ortiz, destacado pensador peruano, solía cuestionar los múltiple problemas morales que afectaban la atención de salud, expresados a través de: la simplificación del acto médico; la negligencia, el abandono, el descuido, la desidia y la indolencia del quehacer galénico; el trato poco profesional, burocrático e inconsecuente otorgado al paciente según el tipo de remuneración económica; los errores diagnósticos con abuso tecnológico, exámenes innecesarios y prescripciones terapéuticas irracionales que, en definitiva, dañaban más que ayudaban al enfermo. Recientemente, tales faltas éticas han sido cuantificadas en nuestro país por Cárdenas quien, analizando las denuncias contra médicos registradas en el Colegio Médico del Perú, en un periodo de 20 años (1991-2010) concluye que el número de faltas éticas consignadas se cuadruplicó y el número de médicos involucrados se triplicó al comparar el periodo 2001-2010 frente al periodo $1991-2000^{(2)}$.

La falta más frecuente al Código de Ética fue "la atención descuidada, superficial, incompleta" (2). Tal hallazgo justifica cuantitativamente una de las tantas razones por las cuales la práctica de la MCP debe incentivarse en el Perú. A ella agregamos otras seis consideraciones que sustentan tal necesidad: El marco conceptual holístico, el paciente en su mundo, el sistema formador, las instituciones de salud, la investigación científica y la responsabilidad del médico.

a) EI marco conceptual holístico. El centro de esta preocupación gira en torno a la pregunta: ¿qué es el hombre? Al respecto, explicaciones de todas las vertientes del saber han sido dadas: filosóficas (3), biológicas ${ }^{(4)}$, económico-filosóficas ${ }^{(5)}$, sociobiológicas ${ }^{(6)}$, religiosas ${ }^{(7)}$ y otras.

En el Perú se ha generado la teoría informacional de la personalidad, de Pedro Ortiz, quien reclama que el hombre no es un animal superior sino un sistema vivo de mayor complejidad evolutiva y, por tanto, de un nivel superno. El Homo sapiens es la única especie que ha desarrollado una superestructura social (la sociedad), la cual se organiza a base de información social que se almacena como cultura y ejerce influencia formativa en todos los seres humanos que la integran. En tal virtud, cada hombre es el producto singular de dos fuerzas de acción, por un lado, epigenética, que expresará las potencialidades biológicas programadas en su genoma $y$, por otro, sociocinética, dependiente del influjo formador de la sociedad cuya información social habrá de registrase en su neocortex. Se forja así, en primer lugar, su conciencia y luego, gradualmente, su personalidad única e irrepetible ${ }^{8-10)}$. Consecuentemente, el hombre será, siempre, producto de su herencia biológica modulada por la sociedad a la que pertenece en interacción constante hasta su muerte.

b). El paciente en su mundo. "El perspectivismo de Ortega y Gasset y su famosa sentencia: Yo soy yo y mi circunstancia, y si no la salvo a ella no me salvo yo, arroja luz en el movimiento de medicina centrada en la persona. Plantea que el hombre trasciende su corporeidad; no solo es él, es él y su mundo (humano o no); y su circunstancia (mundo), no solo influye en él, sino que es parte de él, y él, a su vez, parte de su mundo. La influencia es recíproca. Sin embargo, su mundo no es único. ni rígido Son muchas las circunstancias que rodean al ser en situaciones y roles diversos. Factores naturales y sociales producen variaciones, y siempre, en última instancia, ese mundo personal, en cuanto al significado que cada uno le otorgue, será espacio de apoyo y sosiego o, por el contrario, de estrés, ansiedad y zozobra. En esta óptica, en todo acto médico, nuestra circunstancia será el paciente; por tanto, si no lo cuidamos como circunstancia tampoco estaremos cuidándonos nosotros mismos, como profesionales y como hombres ${ }^{(11)}$.

c). El sistema formador. El concepto de sistema implica un conjunto de elementos interconectados por relaciones de función y dependencia, por lo cual, cada nivel de integración o subsistema es influenciado por los otros niveles ${ }^{(12)}$.

Tal enfoque permite comprender la conducta humana como resultado de la influencia ejercida sobre ella por los diversos niveles del sistema en los cuales el sujeto existe. En tal entendimiento, los niveles del concepto de sistema formador en la educación médica incluyen a la sociedad, la universidad, la facultad de Medicina, las sedes docentes hospitalarias, el cuerpo docente, el cuerpo administrativo y los alumnos. Todos ellos ejercen diversos grados de influencia (formadora o deformadora) en el discente como ser humano individual ${ }^{(13)}$.

Consecuentemente, la influencia que, sobre el desarrollo del estudiante como persona, ejerza la facultad de Medicina, dependerá de la calidad y excelencia de esta. Lamentablemente, en el Perú, la educación superior ha sido invadida por intereses comerciales que han generado una proliferación de facultades de Medicina no siempre dotadas de cuerpos docentes estables y de la calidad necesaria.

En sistemas formadores de pobre calidad aumentará el riesgo de transgredir la verdadera misión de la universidad que, como Gracia apunta, migró de un rol inicial de 
transmisión dogmática e impositiva de los conocimientos de la época, a uno formativo de profesionales y de investigación para crear conocimiento ${ }^{(14)}$. Aunque en el presente, en virtud de lo señalado, la universidad debiera egresar profesionales no solo en nivel de excelencia científica, sino con óptimo desarrollo personal (moral), para asegurar un ejercicio ético profesional que contribuya al bien de la sociedad y al desarrollo del país. Es decir, que la responsabilidad de la educación universitaria, y médica en particular, no debiera considerar al estudiante tan solo como sujeto de aprendizaje profesional, sino como persona para contribuir al desarrollo integral de su potencial.

El sistema educativo debe, asimismo, ser vigilante con respecto a la salud mental del estudiante. Investigaciones realizadas en alumnos de pregrado de Medicina, evidencian diversos problemas de salud mental entre los que preocupantemente destaca el hallazgo de indicadores de conducta disocial ${ }^{(17)}$. Aunque no se está hablando de diagnóstico de personalidad disocial ${ }^{(18)}$, la presencia de tales indicadores apunta al riesgo de un posible desarrollo de tal trastorno en algunos estudiantes. Investigar para intervenir tempranamente es lo que se viene haciendo en estos casos, por medio de procedimientos de tutoría universitaria ${ }^{(19)}$.

d) Las instituciones de salud. Las instituciones de salud, como espacio de prácticas docentes, constituyen elementos importantes en el sistema formador de futuros médicos En ellas, el alumno adquiere las competencias profesionales, no solo por la aplicación de los conocimientos teóricos aprendidos, sino, también, vicariamente, a través del ejemplo de los profesionales que trabajan en tales instituciones. Nadie puede negar la influencia positiva o negativa de tales modelos y de la relación emocional docente-discente en la formación del estudiante.

En tal perspectiva, la ética institucional y la salud organizacional del hospital o puesto de Salud donde ejerza sus prácticas, constituirán importantes climas de influencia, positiva o negativa, en su desarrollo profesional y personal.

Estudios realizados en Perú señalan que las sedes docente hospitalarias no siempre son moralmente los mejores (15,16) y que, si el objetivo educacional incluye ayudar al alumno en su desarrollo como buena persona, aparte de buen profesional, son necesarias las intervenciones correctivas institucionales.

En tal entendimiento, el destino personal y profesional de muchos estudiantes peruanos dependerá de cuán oportuna sea la ayuda que reciban para corregir hábitos y rumbos inadecuados, de modo tal que, al egresar, no solo sean profesionales competentes, sino ciudadanos de bien que contribuyan al desarrollo del país. e) La investigación científica. El intenso desarrollo de la investigación científica en medicina, a no dudarlo, ha generado resultados positivos en cuanto a la comprensión de los mecanismos que subyacen las enfermedades. La medicina basada en evidencias ha impulsado de manera particular la comprensión biológica de la enfermedad ${ }^{(20,21)}$. La MCP, sin embargo, proponiendo una visión más integral del paciente y su mundo, utiliza la mejor evidencia para comprenderlo como totalidad y tratarlo integralmente. En tal propósito, no solo intentará curarlo sino también educarlo en promoción y prevención de su salud. Por ello, plantea que la investigación en Medicina debe estudiar tanto los procesos anormales de la enfermedad cuanto los mecanismos de ajuste hacia ella, así como las potencialidades de desarrollo normal; en otras palabras, la salud negativa y la salud positiva ${ }^{(22)}$.

f) La responsabilidad del médico. Diego Gracia ha planteado claramente este problema. Dice: El término "responsabilidad" es un substantivo abstracto, derivado de otros términos más simples y concretos, a la cabeza de todos, el verbo 'responder'. Ser responsable es ser capaz de responder, tener la capacidad de responder. En principio, no es una característica específicamente humana. Los animales también responden, positiva o negativamente, según el tipo de estímulo que reciben..., pero no tienen responsabilidad. $Y$ esta pequeña, sutil diferencia tiene consecuencias enormes, superlativas. Los animales responden, pero sus respuestas están naturalmente determinadas. En el animal hay por principio una perfecta adaptación natural al medio, ya que en caso contrario desaparece. De las respuestas del animal se puede y debe decir que son 'adaptadas' a su 'medio', pero no que son 'correctas 'o 'responsables'.... el hombre no vive en un mundo puro y estrictamente natural, sino en algo distinto, en un 'mundo' de sentido. Los animales tienen medio; los hombres mundo. El medio es el ámbito de las respuestas naturales; el mundo, por el contrario, es el ámbito de la responsabilidad moral. El hombre tiene, por extraña y asombrosa paradoja, que adaptarse no a un medio sino a un mundo, y para ello sus respuestas no pueden ser meramente naturales, sino que necesitan ser responsables o morales ${ }^{(23)}$.

El médico, como hombre y profesional de servicio, no puede ni debe dejar de ser responsable y ético. Tal actitud y conducta deben estar ínsitamente comprometidas en su vocación, intencionalidad y proceder profesional en la atención de salud de sus pacientes.

En síntesis, la perspectiva peruana sobre la MCP es de real necesidad de promoverla, particularmente en la atención pública. Muy recientemente, el Estado Peruano, a través de su primer ministro, en su presentación ante 
el Congreso de la Republica expresó: Nuestro gobierno abordará los problemas del sector Salud desde un nuevo enfoque: la salud centrada en las personas y no en el enfoque desde el prestador de servicio. En tanto que la ministra de Salud ha incluido en las prioridades del sector el concepto de "rehumanizar la atención de salud centrándola en el paciente" (24).

\section{MEDICINA CENTRADA EN LA PERSONA: UNA PERSPECTIVA DESDE COLOMBIA}

En Colombia, el enfoque de la medicina centrada en la persona (MCP), tiende a ser aceptado con facilidad tanto por los profesionales como por los estudiantes universitarios del campo de la salud. Infortunadamente, tal facilidad es, en muchos casos, engañosa, porque la aparente aceptación no se traduce necesariamente en la aplicación real del concepto en las actividades diarias que lo requieren.

Colombia, sometida desde 1993 a un modelo de salud claramente economicista y neoliberal, puede servir como ejemplo de tal paradoja: mientras la Academia Nacional de Medicina con sus capítulos departamentales, las academias regionales independientes, muchas facultades de Medicina y asociaciones científicas de especialistas, manifiestan abiertamente su aceptación hacia la MCP, las organizaciones intermediarias (empresas promotoras de salud, EPS) y las instituciones prestadoras de servicios de salud (IPS) persisten en considerar "los servicios de salud" como bienes de mercado sujetos a las leyes de la oferta y la demanda, bienes que obtiene no quien los necesita, sino quien puede pagarlos, con lo cual se crea una situación que deforma el actuar de los profesionales introduciendo en su raciocinio la ganancia monetaria como factor implícitamente superior a cualquiera otra consideración. Este panorama se complica más por la exagerada proliferación de facultades de Medicina ocurrida en los últimos veinte años, con más de cincuenta escuelas en actividad y muchas de ellas insuficientemente dotadas en aspectos esenciales de la docencia, como la formación de sus estudiantes en ética, bioética y régimen disciplinario profesional.

\section{El camino de las soluciones}

Preocupada por la situación, y con la creciente evidencia de las actuaciones reñidas con el espíritu de la Medicina, que se producen en todas las regiones de la nación, la Academia Nacional de Medicina comenzó en el año 2012 un gran esfuerzo para buscar el cambio en el sistema de salud; llamó a las principales organizaciones médicas ${ }^{(25)}$ y junto con ellas conformó la
Gran Junta Médica Nacional, cuyo propósito declarado fue lograr el cambio del modelo de atención a la salud; como primer paso, la Gran Junta escribió y propuso al presidente de la República, Juan Manuel Santos, un proyecto de ley estatutaria que declaraba la salud como un derecho humano fundamental, que debía garantizarse a toda persona y que era incompatible con cualquier afán de lucro o pretensión de negocio. El presidente Santos tomó el proyecto con entusiasmo, lo llevó personalmente al Congreso y con su apoyo logró que se convirtiera en Ley Estatutaria, en vigencia desde el 2015. Hace falta, todavía, la reglamentación de aspectos concretos de dicha norma estatutaria, pero ya hay conciencia de que la salud es, en Colombia, un derecho humano fundamental y que en este campo no es admisible ninguna manera de negocio o lucro.

Con esta excelente base, es necesario concretar un complejo proceso que lleve de la aceptación teórica a la completa puesta en acción de la MCP. Tal proceso incluye, entre otras cosas, adherir a una nueva y adecuada definición de la salud, explicar y aceptar las razones científicas que exigen la personalización en este campo, promover los cambios necesarios en la formación de los nuevos profesionales y establecer el modelo de atención en salud que sea compatible con la MCP.

\section{La definición de salud}

En 1999 se propuso en Colombia una nueva manera de definir lo que es salud, a la luz de los conceptos de la teoría caos-complejidad. Al comprender que el ser humano es un sistema hipercomplejo en el cual influyen numerosos "atractores", resulta claro que las respuestas de ese sistema en cualquier terreno, orgánico o mental, son definidamente distintas para cada individuo y no se pueden calcular con precisión matemática, sino verse apenas como probabilidades estadísticas. Al mismo tiempo, cada una de las partes constituyentes del individuo concreto debe tener salud para que, al integrarse, el individuo resultante la tenga a su vez y serán necesarias las características de salud en el ambiente, para que la interacción resulte armónica. Así las cosas, la salud se define ${ }^{(26)}$ como:

Estado vital, dinámico y complejo, caracterizado por adecuado funcionamiento interno y relación armónica con el ambiente.

De lo dicho se desprenden corolarios lógicos: el primero, que la salud debe atenderse con enfoque individualizador, personalizador, sin que ello se considere opuesto a la idea de tener en mente la mejor información científica disponible al analizar las decisiones que se deben tomar. El segundo, que la definición de salud se aplica 
sin dificultad en cualquiera de los órdenes de magnitud del ser humano, desde las moléculas y las membranas hasta los sistemas orgánicos y el individuo completo, así como a las comunidades formadas por esos individuos: familia, sociedad, región, nación y en último término la humanidad toda.

\section{Esfuerzo mancomunado}

Para buscar que la aplicación de la Ley Estatutaria recién conseguida, y el goce del derecho establecido en ella sean realidades que beneficien a todos los colombianos, la Gran Junta Médica sigue planeando acciones concretas para entregarlas como proyectos de leyes ordinarias, donde es necesario, y como marcos de pensamiento para los diversos actores del sistema de salud.

Las distintas facultades de Medicina, las academias regionales de medicina de Caldas y de Cartagena y la Academia Nacional de Medicina han manifestado ya su decisión de adoptar la medicina centrada en la persona y contribuir a su difusión.

Se espera lograr la vigencia completa de la Ley Estatutaria en el curso del año 2016 y cambiar así definitivamente el modelo que en mala hora introdujo las "fuerzas del mercado" y el afán de lucro en las motivaciones para el ejercicio de la medicina y el cuidado de la salud en Colombia.

\section{MEDICINA CENTRADA EN EL PACIENTE. UNA MIRADA DESDE CHILE}

La cercanía entre el médico y su paciente es esencial desde el punto de vista humanitario, pero eso, por importante que sea, no es todo. La información que se puede recoger del paciente y sus familiares acerca de la afección, es diferente según sea en ellos el nivel cultural, la capacidad de observación de lo que ha ocurrido, la carga de prejuicios que cada cual tiene, el grado de compromiso afectivo que traigan y un conjunto de condiciones que pueden hacer que una misma enfermedad aparezca de maneras muy diferentes de un paciente a otro. Esas condiciones solo las puede valorar un médico con una relación próxima al enfermo. Asimismo, al iniciar las terapias, el mayor o menor éxito de estas dependerá, en buena medida, del interés y adhesión a las indicaciones, los que son diferentes de un paciente a otro y, en gran medida, dependientes de la confianza creada entre el médico y su paciente.

Con el ejercicio de la profesión esto se va haciendo cada vez más evidente a los médicos, pero también cada vez más difícil de asumir. La cercanía necesaria entre el médico acogedor y el paciente que busca información y consuelo está hoy día entorpecida por numerosos factores: el escaso tiempo disponible para cada atención médica, la carencia de privacidad en que ocurren muchas de las acciones médicas, el protocolo o guía clínica en que se enmarcan los trabajos clínicos, la hiperespecialización que lleva cada vez más a curar un órgano enfermo que a una persona sufriente, la organización médica que comúnmente asigna un médico al paciente sin que haya habido conocimiento previo entre ellos, la rotativa de médicos que se cambian de turno en turno y el paciente es atendido más por un sistema que por personas, el desarrollo explosivo de la tecnología en la que el médico se valora más por su habilidad en el manejo de un instrumento o en la interpretación de resultados que por la acogida a la persona enferma, etc. Paradójicamente, muchos de estos factores que interfieren en la adecuada relación médico-paciente y han ido deshumanizando la medicina, han permitido que ella sea más eficaz y con mayor cobertura social.

La preocupación por establecer una relación médicopaciente adecuada, se remonta a muchos años. La medicina hipocrática establecía un equilibrio entre enfermedad, paciente y médico lo que ha sido calificado como un descubrimiento cultural de primera magnitud en occidente y relacionado con el nacimiento del diálogo ${ }^{(27)}$. Señalan los Hipocráticos: Es más importante saber qué persona sufre la enfermedad que saber qué enfermedad sufre la persona. En el siglo XII el gran médico y teólogo Maimonides ruega a Dios en su plegaria: Sostén la fuerza de mi corazón, a fin de que siempre esté dispuesto para servir al pobre y al rico, al amigo y al enemigo, al bueno y al malvado. Haz que en el que sufre yo no vea más que al hombre. A comienzos del siglo pasado en tiempos más modernos lo hizo Sir William Osler señalando que cada cual se comporta a su propia manera en la enfermedad y escribía: La variabilidad es la ley de la vida y al igual que no hay dos caras iguales, no hay dos cuerpos iguales, ni dos individuos se comportan igual bajo las condiciones anormales que conocemos como enfermedad". Especialmente se concentró en este tema Francis Peabody, quien en 1927 señaló que los médicos más antiguos se quejan que las generaciones nuevas han recibido mucha enseñanza sobre el mecanismo de las enfermedades y son muy científicos, pero que no saben cuidar a los pacientes, y atribuye esto a la enseñanza que reciben en las escuelas de Medicina que se sienten presionadas a impartir más y más conocimiento en relación al desarrollo del saber médico ${ }^{(28)}$ acotando que: El tratamiento de la enfermedad puede ser enteramente impersonal, pero el cuidado del paciente debe ser completamente personal.

Cabría agregar que cada vez se nota más la falta en la educación médica de humanidades, que no solo 
agreguen cultura a los jóvenes, sino que los sensibilicen para entender mejor a sus pacientes y comprender que detrás de una fisiología alterada hay una persona que está inserta en una sociedad y en una cultura que influyen mucho en su afección.

La preocupación por el respeto de la persona enferma se fue instalando lentamente en la medicina, siendo probablemente una de las primeras manifestaciones de ello la Declaración de la Asociación Médica Mundial sobre "Principios Éticos para las Investigaciones Médicas en Seres Humanos", conocida como Declaración de Helsinki de 1964, la que enfatiza la primacía de los derechos e intereses de la persona por sobre cualquier interés científico.

Probablemente, la publicación de Laine y Davidoff en el Journal of the American Medical Association de 1996 (29) marca el inicio de la medicina centrada en el paciente (MCP) como una corriente que ha impactado en la forma de trabajar, de investigar y de enseñar Medicina apuntando a la persona enferma, con sus valores y creencias, con sus temores, sus tristezas o su alegría, con los objetivos de su vida que puede sentir que se le están frustrando, con sus amores y también sus odios, con su entorno y sus circunstancias. El nombre de MCP ya había sido sugerido por Balint, cuando treinta años antes sugirió la idea de esta forma de hacer medicina ${ }^{(30)}$.

Antes que la medicina alcanzase el nivel de desarrollo actual, las familias tenían un médico general que atendía la enfermedad común y servía de consejero en los problemas sanitarios y, muchas veces, incluso, de orientador para recomendar a los especialistas que pudieran necesitarse. Era el "médico de cabecera" o el "médico de familia". En la organización y complejidad de la medicina actual, ese personaje se echa de menos y debiera promoverse su reinstalación. Él sería el puente para la mejor relación entre los diversos especialistas y la persona enferma y para que esta pueda interactuar con igualdad en las decisiones que se tomen con ella misma aportando uno(s) los conocimientos y el otro sus preocupaciones y necesidades ${ }^{(31)}$.

Un asunto complejo es cómo inculcar el concepto de MCP a los estudiantes de Medicina y a los médicos en general. Esta no es una materia que se imparta solo en el aula. En nuestra experiencia tuvo un fuerte impacto en los estudiantes el presentarles situaciones reales de experiencias clínicas, y hacer un análisis de la información que se le proporcionó al paciente, de las respuestas a sus preguntas, de la participación que tuvieron en las decisiones terapéuticas tanto el paciente como el médico, etc. Estos seminarios se llamaban “¿Y a todo esto, el enfermo qué?”. Trabajos grupales como estos ciertamente que exponen a los estudiantes de Medicina ante temas que suelen caer en el olvido de los docentes y el desconocimiento de los alumnos. Esos seminarios fueron durante un buen tiempo una única oportunidad para que los estudiantes reflexionaran sobre amistad, pudor, respeto, autonomía, dignidad, pena, temor, aspiraciones, intimidad, confidencialidad y otros valores que están muy presentes en la relación médico-paciente y muchas veces se callan, se ocultan o se violentan.

Pero la base para esta enseñanza es aprender de modelos, de tutores que enseñen con su actuar. La enseñanza tutorial es insustituible para el aprendizaje de la clínica y dentro de ese aprendizaje debe incluirse la valoración de la persona enferma. Un buen tutor debe ser, ante todo, buen médico; debe ser estudioso, disciplinado y autocrítico; de buen trato con sus iguales, con el resto del equipo de salud y con sus pacientes; debe interesarse por entender mejor a sus pacientes y comprender que detrás de una fisiología alterada hay una persona que está inserta en una sociedad y en una cultura que influyen mucho en su afección. Lamentablemente, no hay mayor interés de parte de los médicos de asumir la función de tutores, porque los saca de su propia actividad profesional. Las escuelas de Medicina deben buscar formas de atraer a sus mejores egresados para tan importante función.

Es muy importante tener presente que no solo los médicos y otros profesionales de la salud somos responsables de la acogida, consideración y trato digno que se merecen las personas enfermas, las instituciones cumplen un muy importante rol a través de su implementación y organización. Para el paciente no es indiferente los tiempos de espera para la consulta, si en la antesala había una temperatura razonable y se contaba con sillas suficientes, si el establecimiento ofrecía servicios higiénicos suficientes y aseados $y$ condiciones adecuadas para pacientes minusválidos, etc. El objetivo central de la atención médica es el paciente. El médico, el personal de la salud y la arquitectura y el funcionamiento de los establecimientos asistenciales deben adecuarse a las necesidades de los pacientes y no al confort del personal ni a la estética.

Si los establecimientos de salud pueden ser un factor de interferencia en la relación con los pacientes, otro tanto los son otras organizaciones que operan en el sector, como son las aseguradoras, las agencias acreditadoras y otras que han introducido un lenguaje y un pensamiento comercial y colocado al paciente en la condición de clientes o consumidores y a los médicos en la de meros empleados ${ }^{(32)}$. Desgraciadamente, no pocas veces se puede apreciar que los propios médicos se han incorporado al lado comercial dejando atrás una larga tradición y olvidando que su profesión es de servicio. 
Otra importante corriente que ha ingresado al trabajo médico en los últimos decenios, es la medicina basada en evidencias. Esta, a diferencias de la MCP que apunta al paciente como persona individual, se enfoca a lo poblacional, a indagar lo que en estudios grupales parece ser lo mejor para aplicar en el diagnóstico o tratamiento de las afecciones. Como consecuencia de sus resultados, se elaboran protocolos o guías clínicas y se establecen criterios y normas. Ha existido temor que la medicina basada en evidencias se imponga sobre una MCP y sea un factor de deshumanización de la medicina. Lo razonable es que teniendo en consideración las informaciones emanadas de las evidencias, el médico pueda proponer o actuar más informadamente, sin perder de vista que el paciente individual es el centro de su quehacer. Así, la medicina basada en evidencias es un recurso adicional que complementa para hacer aun mejor a la MCP ${ }^{(33)}$.

En el año 2012 se publicó en Chile la Ley 20584 que "Regula los Derechos y los Deberes que Tienen las Personas en Relación con Acciones Vinculadas a sus Atenciones en Salud". Esa Ley tuvo un largo trámite legislativo y ha sido motivo de muchas controversias. Desde luego, es natural que aparezca resistencia a que se legisle sobre dilemas que la medicina no ha resuelto en siglos, y sobre temas complejos y esenciales para los seres humanos en los que hay maneras diferentes de pensar. Además, en alguna medida, esta Ley refleja un grado de resentimiento hacia los médicos, al señalar que las personas tienen el derecho a un trato digno y respetuoso, que los prestadores debemos emplear un lenguaje inteligible durante la atención y que debemos velar porque se adopten actitudes que se ajusten a las normas de cortesía y amabilidad generalmente aceptadas, como si el trato indigno y no respetuoso o el lenguaje inadecuado o la descortesía sean de tanta frecuencia y/o gravedad que se haya hecho necesario no dejarlo más como una actitud básica e implícita y llevarlo a una exigencia legal. Pero la Ley tiene virtudes como es la implantación legal del consentimiento informado y lo regula de manera tal que respete sin duda la autonomía del paciente, y establece la obligatoriedad de que todos los centros cuenten con comités de ética asistencial y norma su funcionamiento. Así, esta Ley ha empoderado a los pacientes y tiende a nivelarlos con el médico, facilitando el diálogo entre ambos. Pero la fuerza de la Ley puede que venza, pero no necesariamente convenza; lo que hay que buscar es que los actores, personal sanitario, pacientes e instituciones, operen sincera y conjuntamente en una MCP. Para ello la Academia Chilena de Medicina realiza seminarios de bioética anuales a los que invita a todos los miembros de los comités de bioética del país, para revisar e inculcar los principales temas de la bioética y así contribuir a tener una medicina ética ${ }^{(34)}$.
En síntesis, la MCP es un estilo de hacer medicina que pretende proteger el aspecto humanitario de la profesión y mitigar los efectos sobre la persona enferma de la medicina tecnificada, comercializada, masificada, funcionarizada, despersonalizada y afectada de múltiples intereses ajenos a los pacientes. Hay que enseñarla a los profesionales de la salud, como forma de trabajo desde el inicio de su contacto con pacientes y quizás la mejor forma de hacerlo, es a través de tutores que sirvan de modelos. No se contrapone con la medicina basada en evidencias, sino que debiera complementarse con ella. Facilita su implementación, contar con médicos de cabecera que pueden ser médicos generales, de familia o especialistas.

\section{PERSPECTIVAS DE LA MEDICINA CENTRADA EN LA PERSONA DESDE EL URUGUAY ASPECTOS FUNDAMENTALES DE LA PRÁCTICA DE LA MCP}

Luego del establecimiento de las bases filosóficas que sustentan la MCP, analizados previamente en un trabajo latinoamericano referencial ${ }^{(35)}$, exponemos los rasgos que singularizan su práctica efectiva útiles para definir con mayor precisión las perspectivas del desarrollo de la MCP en el Uruguay. 1.Construcción de un sistema de salud de enfoque antropocéntrico: centrado en la persona del paciente; 2. Participación efectiva de la persona del paciente en lo atinente a las decisiones de salud que lo puedan involucrar, 3. Visión holística del proceso salud - enfermedad, lo cual lleva a otorgar alta prioridad a las políticas y acciones concretas de promoción y prevención de salud. 4. Humanismo creciente de la práctica médica, en coincidencia con los propios objetivos de la bioética clínica,

\section{FACTORES RELEVANTES VINCULADOS AL DESARROLLO DE LA MCP EN EL URUGUAY}

Como fortalezas y desde una concepción humanista, el Uruguay ha estructurado un sólido marco jurídico como sustento de un sistema nacional integrado de salud (36), con reconocimiento pleno de los derechos de los ciudadanos en lo relativo al logro de una salud integral ${ }^{(37,38)}$. Cuestión aparte será determinar el grado de aplicación real de estos fundamentos jurídicos, lo que constituye en el Uruguay, motivo permanente de discusión política y social, pero que se erige en importante estímulo práctico para la búsqueda de soluciones, aun cuando estas últimas sean inevitablemente parciales y no sistematizadas. 
Ha sido una tradición permanente, por parte de la sociedad uruguaya, la búsqueda de la equidad en el acceso y provisión de las prestaciones asistenciales. Un ejemplo paradigmático que abona la precedente afirmación se halla representado por la conformación, desde 1980, de un organismo denominado Fondo Nacional de Recursos que, con un aporte social mínimo, pero universal, ha permitido a cualquier ciudadano, con prescindencia de sus recursos personales, acceder a las prestaciones propias de la medicina altamente especializada.

En contraparte, como debilidades más significativas, podemos mencionar una sostenida carencia de recursos económicos propios y de manejo autónomo no derivados hacia el nivel central, y que permitan sostener inversiones y gastos limitados en tanto insumos locales. Los mencionados insumos serán esenciales para el medio, a condición de que los mismos puedan aplicarse sin mayores demoras, es decir, sin depender de la habitual "marcha burocrática" de su retorno parcial.

Esta ausencia de descentralización operativa y económica, un rasgo de impronta histórica típica de aquellos países latinoamericanos que, como el Uruguay, se caracterizan por su fuerte estructura centralizada es, en parte, responsable de mantener sus diferentes regiones con marcados atrasos relativos entre sí y de todas ellas con la ciudad capital. Asimismo, este factor explica la lentitud en el ritmo de inversiones para instalar un programa permanente de mejoras de la infraestructura sanitaria y, posiblemente, colabore, en la persistencia de situaciones de flagrante inequidad, sobre todo las de origen geográfico, cuya magnitud se halla en relación directa con la distancia a la capital del país del eventual afectado. Este factor hace sentir todo su impacto en la respuesta asistencial frecuentemente tardía a las denominadas patologías tiempo-dependientes: accidente cerebrovascular o infarto agudo de miocardio, entre otras.

La exigencia real por parte de la autoridad sanitaria nacional sobre el cumplimiento de las disposiciones legales vigentes, ofrece un panorama contradictorio. En efecto, dicho contralor se halla ausente (o resulta actualmente muy incipiente) en la verificación del grado real de funcionamiento de los comités de ética asistencial, pero no en las formalidades de su integración. Por otra parte, en el momento actual, se está procesando la incorporación de los representantes de la comunidad en los órganos de gobierno de las instituciones asistenciales del denominado sector privado.

Apuntando al avance efectivo de la MCP, entendemos que este involucramiento de los usuarios en la gestión del modelo asistencial se halla positivamente orientado hacia la consolidación del paradigma ya citado, a condición de que se universalice dicha participación.

La sempiterna ausencia de una práctica transparente y efectiva de acreditación institucional, ha impedido, entre otras cosas, avanzar hacia una racionalización del traslado sanitario que permita derivar cualquier situación clínica hacia el centro más cercano en condiciones de resolver la situación patológica, de acuerdo al nivel de complejidad de esta. Lo precedente significa sostener el principio operativo del aumento en la resolutividad local, sin trasladarla al nivel central. Por otra parte, en casi todo el territorio se carece de una regionalización planificada que posibilite una derivación específica diferente a aquella vinculada a la capital del país. En consecuencia, como resultado de ambas ausencias, acreditación y regionalización, la ciudad capital se vuelve destino inevitable de recepción de la inmensa mayoría de los citados traslados. Como resultado, los mismos son efectivizados con sensibles demoras resolutivas, desarrollo de patología intrínseca al acto médico del traslado y sobrecarga de los centros de referencia terciaria. Por otra parte, resulta muy lento el avance de una coordinación efectiva entre los sectores público y privado, con la consiguiente duplicación de servicios e incremento de costos totales del sistema.

Sin embargo, en el momento actual se abren numerosas oportunidades, sobre cuya concreción deberá insistirse, a fin de acelerar la marcha del proceso de consolidación de la MCP.

En los últimos años se ha iniciado, liderado por el Colegio Médico del Uruguay ${ }^{(39)}$, el proceso de recertificación profesional, de inicio voluntario y concebido en términos tales como para asegurar la mejor formación científico técnica y ética de sus médicos. Esta concepción resulta equivalente a tratar de establecer un nuevo y más garantista contrato social entre la profesión médica y la sociedad uruguaya en su conjunto.

El progresivo incremento de la participación ciudadana en la determinación del diagnóstico de necesidades y en las decisiones de políticas de salud de la comunidad, se halla en concordancia con la filosofía de la MCP, al mismo tiempo que facilita la implementación de la misma. Un caso específico de esta situación, y que nos interesa de manera especial, por tratarse de órganos de resolución de conflictos ético - clínicos, es el que resulta de la integración de los usuarios en los comités de ética asistencial la cual, aun siendo escasa en el momento actual, viene mostrando una clara tendencia a su incremento progresivo.

Por otra parte, el Código de Ética Médica en el Uruguay tiene categoría de Ley de la Nación ${ }^{(40)}$. Desde el punto 
de vista de la vigilancia ética del ejercicio profesional, este rango legal la hace ingresar en una situación contradictoria. En efecto, por un lado, se refuerza la observancia de los fundamentos éticos del ejercicio profesional, puesto que cualquier violación de su articulado constituye, a la vez, una falta ética y una violación de una norma legal. Sin embargo, dicho rango legal hace correr el riesgo de terminar judicializando cualquier falta ética que es, justamente, lo que quería evitarse con la aplicación del código, al mismo tiempo que dificulta llevar a su término cualquier futuro intento de actualización del mismo, puesto que hay que cambiar nada menos que una Ley de la Nación.

Resulta imprescindible procesar con dos instancias, una plebiscitaria en el cuerpo médico y otra posterior, parlamentaria, y con el apoyo de la pertinente política educativa, una serie de cambios culturales en la aplicación efectiva de los principios bioéticos, cuyo análisis detallado no es posible aquí. Sin embargo, y a manera de síntesis, se intentará resumir este punto en una serie de enunciados, expuestos a continuación.

A nivel del principio de autonomía, es necesario transitar desde la información pretendidamente exhaustiva e imposible de alcanzar plenamente hacia la participación efectiva de la persona del paciente en la elaboración de su propio consentimiento informado.

En términos actuales, si bien la propuesta beneficente para una determinada situación de enfermedad la debe elaborar el equipo tratante, con base en la mejor evidencia disponible, la decisión beneficente será aquella que finalmente determine el paciente, "esclarecidamente" informado. La ausencia de repercusiones maleficentes de una determinada terapéutica deberá prevenirse específicamente, pero, además, se hace necesario instrumentar de manera sistemática una política de seguridad del paciente a nivel institucional y del propio sistema de salud.

Los criterios de justicia distributiva no debieran utilizarse como un elemento sustancial para incidir en la toma de decisiones para su aplicación vinculante respecto de una persona o situación clínica en particular, tal como nos lo ha enseñado Diego Gracia (41). Aquella situación de justicia distributiva deberá alcanzarse, más bien, como consecuencia de las decisiones acertadas de macrogestión sanitaria antes que por el sacrificio de las peticiones individuales, cuando estas últimas sean legítimas y se hallen bien fundamentadas por las mejores evidencias científicas disponibles.

La persona enferma es siempre vulnerable, y tal circunstancia se debe tener en cuenta estableciendo, en circunstancias en las que existan limitaciones propias o riesgos intrínsecos aumentados y en acuerdo con la convicción autonómica legítima de la persona del paciente, los correspondientes recaudos de protección integrantes de la ética del cuidado.

La dignidad del ser humano debe incluirse en toda propuesta de decisión, en la medida en que su debida preservación mantiene incólume el núcleo esencial de la propia humanidad de la persona enferma. De esta forma, esta dignidad preservada se convertirá naturalmente en una parte fundamental y proactiva del propio proceso de recuperación.

Por último, entendemos trascendente incorporar en los planes de estudio del futuro profesional de la salud, la moralización formativa, tal como ha sido propuesta por Perales ${ }^{(42)}$, puesto que la misma procuraría que los egresados, además de su excelencia técnica y ética, fueran también mejores y más solidarias personas, dignos practicantes de una profesión de servicio.

\section{PERSPECTIVAS Y DESAFÍOS}

Colocar a la persona del paciente en el centro de los actuales sistemas de salud significa llevar a cabo las imprescindibles reformas estructurales y funcionales de las instituciones y de los propios sistemas de salud que esta concepción antropocéntrica implica. Resulta casi obvio señalar el profundo cambio cultural que este programa de rediseño que se enuncia puede significar $y$, por ende, incidir en las correspondientes acciones educativas vinculantes. Uruguay ha elaborado una formulación doctrinaria acorde con los principios de la MCP y plasmada en un corpus jurídico coherente, existiendo ya un considerable camino recorrido en la concreción de un sustancial cambio de modelo. Corresponde, ahora, profundizar la reforma, lo cual es posible mediante acciones que se hallen alineadas con los principios que sustentan la práctica de la MCP.

Para finalizar, y a manera de síntesis, las perspectivas de la consolidación de una MCP en el Uruguay parecen favorables, en la medida en que el país se halla transitando una etapa avanzada de cambio de modelo asistencial, en consonancia con los principios de la Bioética y los postulados de un nuevo paradigma humanista como el representado por la MCP, la cual se propone volver a colocar a la persona en el centro de las políticas de salud actuales.

\section{CONCLUSIONES}

Las diferentes perspectivas expuestas en el presente trabajo coinciden en destacar la necesidad actual de incluir en los programas de atención de salud de la región el enfoque de medicina centrada en la persona, tanto para proteger su esencia humanitaria cuando se aplica al hombre en situación de enfermedad, cuanto 
comprometida con su desarrollo integral en situación de salud, y proteger también a la población de la influencia nociva de las demandas del mercado apoyadas en el erróneo concepto de la salud como bien de consumo. Tal proceso parece perfilarse con buenas posibilidades en Latinoamérica, dado el hecho de que varios países vienen emprendiendo procesos de reforma estructural en sus sectores de salud.

Finalmente, para cumplir con estos objetivos, debemos destacar la necesidad de la MCP de generar mayor conocimiento por medio de la investigación científica, extendiéndola no solo al paciente como persona sino, también, a la persona del profesional en ejercicio y a los estudiantes en formación, buscando equilibrar las variables de ciencia y humanismo en la educación médica y de salud en general.

Conflictos de interés: los autores declaran la ausencia de todo conflicto de interés en relación con el desarrollo de este trabajo.

Fuentes de financiamiento: autofinanciado

\section{REFERENCIAS BIBLIOGRÁFICAS}

1. Mezzich JE, Kirisici L, Salloum IM. ICPCM Project on the Systematic Conceptualization and Measurement of Person- and People-centered Care Technical Report, New York: International College of Person Centered Medicine, 2014.

2. Cárdenas M.El daño medico: su regulación ética y legal en el Perú. Tesis de Doctorado en Medicina. UNMSM. Unidad de Posgrado. 2016. (en trámite)

3. Muñoz -Pérez E. Heidegger y la pregunta por el hombre. VERITAS, 2007, II, 16:91105

4. Campbell N A. Biology. Third Edition. The Benjamin/Cummings Publishing Company, Inc. California, 1993

5. Erich Fromm. Marx y su concepto del hombre. Karl Marx: Manuscritos económico-filosóficos. Breviarios. Fondo de Cultura Económica. México1962.

6. Wilson E O. Sociobiology: The New Synthesis. Cambridge, MA. Harvard University Press, 1973

7. Sociedad Bíblica Católica Internacional. La biblia latinoamericana. Verbo Divino. Madrid, 1972

8. Ortiz P. La Formación de la Personalidad. Lima: Dimaso E.I.R.L; 1997

9. Ortiz P. Introducción a una psicobiología del Hombre. (Capítulo 7. Estructura de la actividad Personal) Cuadernos de Psicobiología Social 1. Universidad Nacional Mayor de San Marcos, Lima, 2004

10. Ortiz P. Educación y formación de la personalidad Fondo Editorial UCH. Lima, 2008.

11. Perales A. Calidad de la Atención Médica: aspectos éticos. En León Barúa R. Calidad dela Atención Médica. Academia Nacional de Medicina. Serie: Problemática Sanitaria Nacional: opinión institucional. Lima, 2015.

12. Bertalanffy Von Ludwig. Teoría General de los Sistemas. Tercera reimpresión. Fondo de Cultura Económica. México, 1992

13. Perales A. Ética y humanismo en la formación médica. Acta Bioética, 2008, 14 (1): $30-38$

14. Gracia D, Construyendo valores. Triacastela. Madrid. 2013.

15. Perales A, Ortiz P, Norberto V. Estado de moralidad/inmoralidad de una escuela de medicina y un hospital público: aplicación de una estrategia de moralización. An Fac med. 2008;69(2):97-103

16. Perales A. Ética institucional y estrés laboral. Acta Médica Peruana, 2008, 25 (1): 50-51
17. Perales A, Alarcón J, Sánchez E, Arcaya MJ, Cortez E, Parhuana A, Caballero J, Carrera R, Torres H. Conducta suicida en estudiantes de medicina de una universidad nacional peruana. En, Violencia y Trauma en el Perú. Desafíos y Respuestas. I Bustamante, M Rivera, L Retamozo (Eds) Universidad Peruana Cayetano Heredia, Lima, 2013

18. OMS. CIE 10. Trastornos Mentales y del Comportamiento. Descripciones clínicas y pautas para el diagnóstico. Técnicas Gráficas FORMA S.A. Madrid. 1992.

19. Saavedra C, Perales A, Oliveros M, Zavala S, Izaguirre M. Manual de Tutoría Universitaria. UNMSM. Lima, 2015

20. M. Vega-de Céniga, N. Allegue-Allegue, S. Bellmunt-Montoya, C. Lopez-Espada, R. Riera-Vázquez, 'T. Solanich-Valldaura, J. Pardo-Pardo. Medicina basada en la evidencia: concepto y aplicación. ANGIOLOGÍA, 2009; 61(1): 29-34

21. Soto A. Ensayos clínicos y salud pública en el Perú: reconciliando un innecesario divorcio. Rev Peru Med Exp Salud Pública. 2012;29(4):429-30.

22. Saavedra J. Estudios epidemiológicos centrados en la persona y la comunidad: perspectivas comunitarias de personas con problemas de salud mental en zonas rurales del Perú. Rev Peru Med Exp Salud Pública, 2016 (en prensa)

23. Gracia D. Profesión Médica. Investigación y justicia sanitaria. ética y vida. Estudios de Bioética 4. Editorial El Buho Ltda. Bogotá, 1998

24. Frazer B. Peru's new Health minister outlines priotities. The Lancet. August 19 2016. DOI: http://dx.doi.org/10.1016/ S0140-6736(16)31415-5

25. Federación Médica Colombiana, Colegio Médico Colombiano, Asociación Colombiana de Sociedades Científicas, Asociación Médica Sindical Asmedas, Asociación de Médicos Residentes e Internos, inicialmente. Después se unieron la Asociación de Estudiantes de Medicina, la Asociación Colombiana de Facultades de Medicina ASCOFAME.

26. Mendoza-Vega J. La Salud, un "fractal" abstracto. Bases para una propuesta de definición. Rev Medicina vol. 20 (2) (48), Bogotá 1998

27. Velásquez G O en Presentación del Libro la Herencia de Hipócrates de Alejandro Goic. Bol de la Academia Chilena de Medicina 2014;51:337-349

28. Peabody FW The Care of the Patient JAMA 1927; 88:877-882
29. Laine C, Davidoff F. Patient-centered medicine. A professiona l evolution JAMA 1996; 275:152-6

30. Balint E. The possibilities of patientcentered medicine. J R Coll Gen Pract 1969;17:269-76.

31. Bardes CL Defining "Patient-Centered Medicine" ., M.D. N Engl J Med 2012. 366:782-83

32. Glass RM. The Patient-Physician Relationship Focuses on the Center of Medicine. JAMA 1996;175:147-48

33. Sacristán JA. Medicina basada en la evidencia y medicina centrada en el paciente: algunas reflexiones sobre su integración. Revista Clínica Española 2013; 213:460-464

34. Bravo M. Reflexiones sobre Bioetica: Seminarios de la Academia Chilena de Medicina (2011-2013). Academia Chilena de Medicina 2015

35. Wagner P, Perales A, Armas R et al: Bases y perspectivas latinoamericanas sobre medicina y salud centradas en la persona. An Fac Med. 2015; 76(1):63-70.

36. Ley 18.211 del Sistema Nacional Integrado de Salud. Accesible el 4 de setiembre 2016 en: www.msp.gub.uy/sites/default/ files/18.211.pdf

37. Ley 18.335 de Derechos y Deberes de los Pacientes. Accesible en pdf el 4 de setiembre 2016 en: www.psico.edu.uy/sites/ default/files/reglamentacion-derechosusuarios-2010.pdf

38. Decreto Ministerial 274/010 Reglamentación de la Ley 18.335 Accesible en pdf el 4 de setiembre 2016 en: www.diputados.gub.uy/wp-content/ uploads/2014/11/d3680.pdf

39. Ley de Colegiación Médica. Accesible el 4 de setiembre 2016 en: https://parlamento. gub.uy/documentosyleyes/leyes/ ley/18591

40. Código de Ética Médica: Ley 19.286. Accesible el 4 de setiembre 2016 en: www. colegiomedico.com.uy/Home.aspx

41. Gracia, Diego: Fundamentos de la Bioética. Madrid. Triacastela. 2008.

42. Perales A. Educación médica en Latinoamérica centrada en la persona Presentado en la Reunión de Academias Latinoamericanas de Medicina, Lima, 13 diciembre, 2014.

Correspondencia: Alberto Perales Cabrera Correo electrónico:perales.alberto@gmail.com 